\title{
MoviLetrando: Jogo de Movimentos para Alfabetizar Crianças com Down
}

\author{
Emanuel Henrique Farias ${ }^{1}$, Marcelo da Silva Hounsell ${ }^{1}$, Luziane Bombazar \\ Blume $^{2}$, Francine Rohrbache Ott ${ }^{2}$, Fernanda Vianna Pinto Cordovil ${ }^{2}$ \\ ${ }^{1}$ LARVA - Laboratory for Research on Visual Applications, DCC - Departamento de \\ Ciência da Computação, UDESC - Universidade do Estado de Santa Catarina. \\ Joinville - SC- Brasil \\ ${ }^{2}$ NAIPE - Núcleo de Assistência Integral ao Paciente Especial, PMJ - Prefeitura \\ Municipal de Joinville. \\ Joinville - SC - Brasil \\ emanuelfarias@outlook.com,marcelo@joinville.udesc.br, \\ fvpcm@hotmail.com,franfisio@ig.com.br,luziane.blume@ig.com.br
}

\begin{abstract}
Games have been developed with purposes beyond the scope of simple entertainment, leading to what has been called Serious Games (SG). $S G$ have shown efficacy in many areas of knowledge. But all the benefits of SG have been underexplored by a population that lacks much stimulus in the teaching-learning process: the Intellectually Disabled. Stimulating motor and cognitive functions can be beneficial to this population, specialliy if no special and expensive devices are needed. This paper presents a $S G$ for children with Down syndrome that stimulates motor (through movement) and cognitive functions aiming at the literacy, but using webcams only.
\end{abstract}

Resumo. Recentemente os jogos têm sido desenvolvidos com propósitos além do entretenimento, levando ao que se chama de Jogos Sérios (JS). Os JS têm mostrado eficácia em várias áreas do conhecimento. Mas, todos os benefícios dos JS têm sido pouco explorados em uma população que carece muito de estímulo no processo ensino-aprendizagem: os Deficientes Intelectuais. Estimular as funções motoras e cognitivas com JS pode ser benéfico para esta população, ainda mais se usar uma tecnologia que evite o uso de dispositivos especiais elou caros. Este artigo apresenta um JS para crianças com síndrome de Down com o objetivo de estimular as funções motoras (através do movimento) e cognitivas com vistas à alfabetização, usando só uma webcam.

\section{Introdução}

Segundo o Censo Demográfico de 2010, 23,9\% da população brasileira declarou ter pelo menos uma deficiência, sendo $1,4 \%$ mental/intelectual. É possível, fazer uma estimativa baseada no fato de que 1 para cada 700 ou 1000 nascimentos vivos (ROIZEN, 2002) apresenta Síndrome de Down (SD). "Com esses dados estima-se que cerca de 270 mil pessoas no Brasil teriam SD” (op.cit.).

Apesar de não se saber exatamente quantos destes apresentam a SD, sabe-se que a característica da alteração cromossômica que define a SD, não significa que todas 
apresentam o mesmo quadro, mas sempre, SD é caracterizada por Déficit Intelectual (FSD, 2012). Por causa disso, há um atraso na aquisição das funções motoras, de linguagem, afetiva, e operacionais mas, não uma impossibilidade ou limitação de atingilas, o que portanto, requer estimulação específica e mais intensificada como forma de desenvolvimento.

Na deficiência mental o funcionamento intelectual é significativamente inferior à média. "O aluno com deficiência mental tem dificuldade de construir conhecimento como os demais e de demonstrar a sua capacidade cognitiva" (GOMES et. al., 2007, p.16). Mesmo durante tarefas de aprendizagem e/ou entretenimento, as crianças com SD precisam de estímulo para produzirem movimentos cada vez mais precisos pois "todas as crianças executam movimentos desastrados e imaturos no começo e vão refinando seus movimentos com a prática" (SACKS; BUCKLEY, 2003, p. 135). A prática melhora tanto a velocidade dos movimentos quanto a precisão dos mesmos (op. cit., p. 137). Portanto, prática e movimentos devem ser incluídos em todas as atividades possíveis.

Um importante passo na vida de uma pessoa qualquer, e em especial a com SD, é a alfabetização. Um novo mundo de informações surge possibilitando independência em suas atividades, facilitando a inserção no mercado de trabalho, trazendo assim, mais autonomia financeira para a pessoa e favorecendo também a sua família.

Jogos são considerados a mídia mais apropriada para o novo paradigma educacional da geração dos Nativos Digitais (PRENSKY, 2001)(DESHPANDE; HUANG, 2009). Os denominados "Jogos Sérios" (JS) aparecem a medida que o objetivo principal do jogo deixa de ser o entretenimento mas, algo mais sério, como o combate ao sedentarismo, por exemplo - mesmo que o jogador ainda ache que é entretenimento. Os JS enfatizam vários aspectos como: sociabilidade, atividade física, desenvolvimento da atenção, memória e raciocínio lógico (dentre outros), possibilitando um mecanismo lúdico de promoção da qualidade de vida. Assim, é de se imaginar que este tipo de software (os JS) possam ser usados no desenvolvimento de pessoas com deficiência intelectual, como as pessoas com SD.

Rizzo (2001) afirmou que a Realidade Virtual (RV) é uma "ferramenta útil para o estudo, avaliação e reabilitação de processos cognitivos e atividades funcionais" e constatou que a capacidade da RV de estimular e armazenar respostas oferece oportunidades de avaliação e reabilitação clínica que não se tem disponível com métodos tradicionais e cunhou o termo Reabilitação Virtual. A Reabilitação Virtual trata das aplicações, características, benefícios e desafios do uso da RV na reabilitação, até porque já foi identificado que o exato impacto dos procedimentos tradicionais de reabilitação é obscuro e incerto (CAMEIRÃO et al., 2007).

Existe um tipo de RV (SHERMAN \& CRAIG, 2003) denominado de "RV de Projeção", onde a interação com o usuário é capturada por câmeras (webcam) permitindo que os movimentos e ações do usuário interajam com um mundo virtual. Ou seja, usam recursos de Interfaces Naturais. Já existem jogos de computadores que usam webcams mas nem todo jogo por computador que usa webcam são exemplos de RV de Projeção pois, estes últimos permitem ao usuário ver sua imagem dentro do ambiente virtual/jogo. 
Um JS que utiliza recursos de RV de Projeção e Interface Natual usa uma metáfora de espelho (ou visão em segunda pessoa (ot. cit.)) e se mostra apropriado para o desenvolvimento de jogos para pessoas com SD pois as pesquisas indicam que estas pessoas dependem mais de feedback visual quando executam uma tarefa do que indivíduos típicos (SACKS \& BUCKLEY, 2003, p. 137). Usando essa mesma técnica de projeção, o player consegue interagir com o jogo usando movimentos, assim, é possível estimular o lado motor.

Pode-se usar um jogo comercial para ter uso educativo (aplicações educativas desenvolvem: atenção, colaboração, memória, etc.) mas, aqueles jogos cujos desenvolvimentos foram direcionados para um determinado fim tem, certamente, um potencial maior de alcançar o seu objetivo. Deste modo, desenvolver JS com recursos da RV de Projeção e Interface Natural para SD resulta numa atividade que pode ser lúdica, repleta de feedback, ajustada a cada pessoa e sem necessitar do uso de dispositivos de controle repletos de botões e sequencias complicadas de acionamento e ajudar na aquisição de habilidades cognitivas (como a alfabetização) que possam ajuda-la a aumentar sua independência ou até autonomia financeira.

Portanto, os seguintes questionamentos se mostram importantes: é possível promover o início do processo de alfabetização em crianças com SD usando um JS? Uma mecânica de jogo com tecnologia com uma metáfora de espelho ajuda no desenvolvimento motor? A conjunção de dois aspectos (cognitivo e motor) criando uma interface de dupla tarefa beneficia os dois aspectos ou dificulta? Para iniciar na investigação dos questionamentos acima, este trabalho propõe um JS que visa ensinar crianças com SD usando tecnologia de RV de Projeção.

\section{Fundamentos}

\subsection{Alfabetização}

Durante o processo de alfabetização, ou letramento, as crianças passam, normalmente, por quatro etapas (SANTAMARIA, p. 238). A primeira, a pré-silábica, onde a criança começa a perceber e reconhecer o formato das letras e dos números, conseguindo diferenciá-los. Também consegue reproduzir traços parecidos com os do nosso alfabeto. $\mathrm{Na}$ segunda etapa, chamada silábica, a criança já consegue escrever as letras corretamente. Nesta etapa a criança começa a perceber as sílabas nas palavras e ainda contar a quantidade de letras e sílabas na palavra. Esta etapa também se caracteriza pela relação entre a escrita e seu fonema. No desenvolvimento desta etapa a criança começa a ler sílaba por sílaba das palavras, assim, a palavra deixa de ser entendida apenas como um desenho. A terceira fase, silábico-alfabético, a criança descobre que a sílaba pode ser composta por unidades ainda menores. Na quarta fase a criança começa a ter uma escrita mais regular, e uma percepção fonológica das palavras.

Portanto, todo o processo de letramento inicia-se pelo desenvolvimento da capacidade de identificar e distinguir os desenhos e rabiscos que então, passarão mais tarde a obter um significado sonoro.

Portanto, prática e movimentos devem ser incluídos em todas as atividades possíveis. Vale lembrar que as habilidades motoras e cognitivas de uma criança com SD 
é basicamente atrasada e não comprometida (op. cit., p.138), o que sugere benefício de maior estimulação e mais prática, para encurtar esse atraso.

\subsection{Jogos Sérios}

Jogos Sérios (JS), por sua vez, são uma recente vertente dos jogos computacionais definidos como jogos principalmente focados nos aspectos educacionais e não no entretenimento (MILLER et al. 2001). Girard sustenta a ideia de que Jogos Sérios não são jogos diferentes mas, são diferentes de jogos, outra categoria de aplicações computacionais. Já Marsh (2011) defende que JS são um subconjunto especial de jogos de videogame que tem um propósito de utilidade. Mas o que se pode perceber de consenso entre os autores é o fato de que um resultado específico e sério se espera desses jogos, não é só laser. Espera-se conhecimento, reabilitação, desenvolvimento, movimento, dentre outros benefícios.

Se não pela efetividade direta do JS pode promover, uma vez que esta ainda precisa ser comprovada cientificamente para cada caso, já existem evidencias quanto ao aumento do envolvimento (ANNETTA et al., 2010) e motivação (BAKER et al., 2010) com uso de JS o que incorre em treinamentos por longos períodos de tempo e já foram sugeridos também para aumentar estes aspectos e também a atenção de crianças com déficits de atenção (ANNETTA et al., 2010).

\section{Trabalhos Relacionados: Jogos Sérios para Populações Especiais}

O uso de jogos como recurso educacional já foi indicado como uma tendência tecnológica (MARTIN et al., 2011). Mas, em função das necessidades e especificidades de crianças com DI, essa tendência ainda não chegou a elas. E esta especificidade precisa ser contemplada nos JS apropriados para este público também pois, sem especificidade, nem mesmo crianças normais percebem impacto nas suas vidas (FARRINGTON, 2011).

BURKE et al. (2008) analisaram jogos comerciais e sistemas já existentes utilizados para a reabilitação motora. Jogos existentes, baseados nos sistemas comerciais EyeToy (2012) e Gesture Extreme (GX, 2012) foram descritos como apresentando o principal problema que é a não adaptação das tarefas do jogo para as limitações específicas do paciente, o que acarreta na possibilidade de um jogo exigir que um paciente realize uma tarefa que ele não tem condições físicas efetivas de realizar e portanto, causando frustração.

Um jogo modificado para uso por crianças com paralisia cerebral (MERIANS \& FLUET, 2011 apud RIZZO 2012) com práticas de uma hora por dia, três vezes por semana, por três semanas, jogaram 3 ou 4 jogos adaptados e foram submetidos a testes clínicos e cinemáticos. Testes clínicos incluiram o MAUULF (Melbourne Assessment of Unilateral Upper Limb Funcion) que mede a qualidade do movimento de 16 atividades com extremidades dos membros superiores e, testes cinemáticos incluíram a velocidade do braço, suavidade da trajetória da extremidade. Este trabalho mostrou que as crianças com o treinamento apresentaram uma trajetória mais direta para tentar tocar os 10 objetos em um dos jogos. Também, as crianças melhoraram a suavidade da trajetória quando tentam alcançar certos objetos e houve uma melhora estatisticamente significativa no MAUULF após o treino. 
O Dance2Rehab (BRUCKHEIMER, HOUNSELL e KEMCZINSKI, 2010) é um jogo que estimula tanto os aspectos motores quanto cognitivos. O estímulo motor é obtido pelo uso da metáfora do espelho onde imagem do jogador é capturada por uma webcam e o jogador é colocado dentro do ambiente virtual, podendo interagir com os objetos que o computador gera/controla. Desta forma, a tela se comporta como um espelho onde o jogador fica se observando.

O estímulo cognitivo é obtido pelas regras de funcionamento do jogo ao qual a criança deve perceber para poder pontuar, como exemplo, a mudança de cor de um objeto em uma determinada posição da tela permite que o player, ao encostar no objeto, pontue mais. Este jogo foi desenvolvido em parceria com fisioterapeutas, especialmente para ser usado por crianças com síndrome de Down e obteve resultados significativos no desenvolvimento motor.

Concluindo, a população com SD, representa uma pequena mas não desprezível parcela da população que requer uma atenção maior nos aspectos educacionais (motor e cognitivo) afim de se tornarem cidadãos produtivos. O uso de JS com tecnologias de RV de Projeção parece ser o tipo de software apropriado capaz de promover estímulo e adaptabilidade e, ao mesmo tempo, entretenimento e ludicidade.

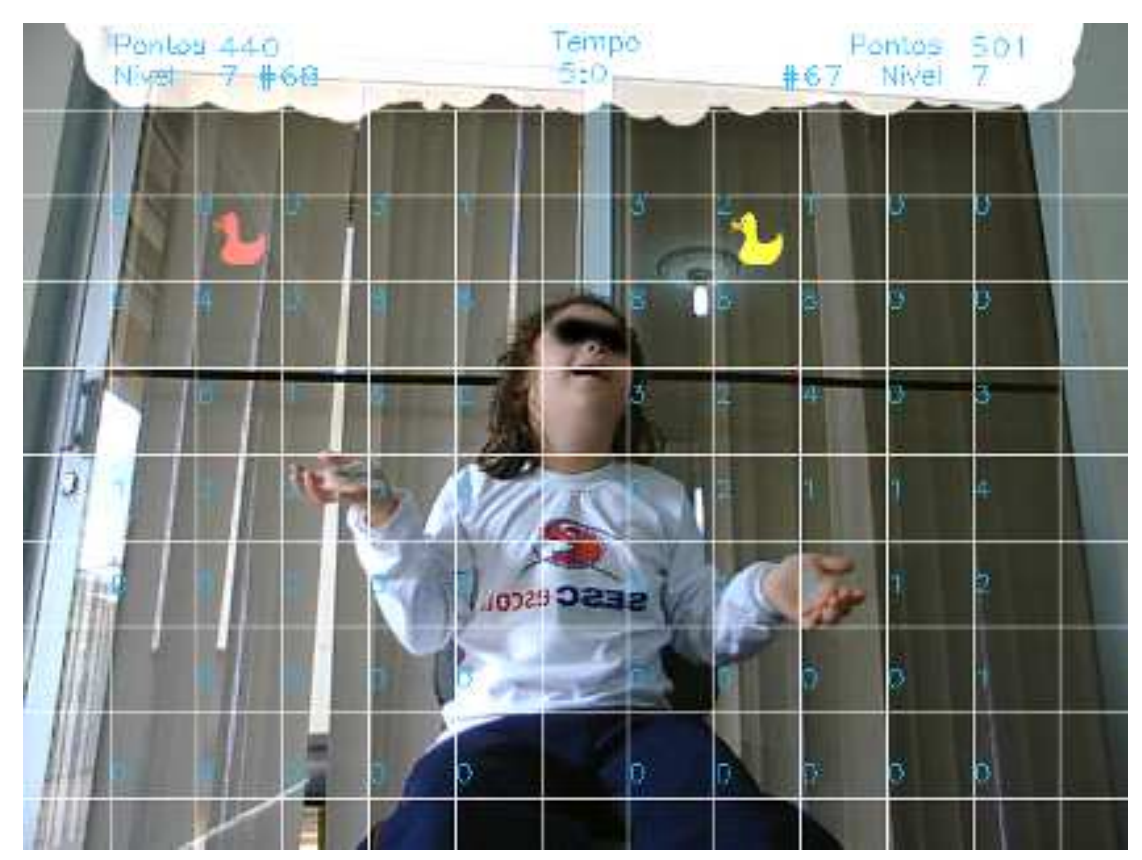

Figura 1: Criança com Down jogando o Dance2Rehab

Entretanto, deve-se questionar quais são os recursos e características de design que tais softwares devem apresentar e qual a efetividade que ele traz no desenvolvimento motor, funcional e cognitivo (onde se insere o processo de alfabetização) para as pessoas com SD?

\section{O MoviLetrando: Aprendendo Letramento com Movimento}

O jogo MoviLetrando tem como público alvo crianças com Síndrome de Down (SD) e como objetivo, dar os primeiros passos no letramento dessas crianças. Para que seja 
possível essa evolução, é necessário também, que algum profissional da área da saúde, esteja acompanhando as fases em que a criança está jogando. O jogo está sendo desenvolvido no LARVA (LAboratory for Research on Visual Applications), laboratório de pesquisa da UDESC. É fruto de uma equipe de desenvolvimento multidisciplinar composta por duas pessoas da área da computação, duas fisioterapeutas e uma psicóloga. O MoviLetrando usa o conceito de RV de Projeção, de Jogo com Movimento e sem dispositivo de interação para facilitar a usabilidade. Uma tela do meio do jogo é mostrada na Figura 2 onde podem ser observados vários elementos (descritos a seguir).

Para jogar o MoviLetrando é necessário uma webcam e um PC convencionais. A imagem capturada pela câmera é o próprio jogador e o cenário do jogo. Este aspecto ajuda a desenvolver a propriocepção, que é a capacidade do indivíduo conhecer o próprio corpo e definir as estratégias motoras necessárias para executar um determinado movimento.

Quando o jogo inicia, efetua-se a calibração: aparece na tela uma silhueta onde a pessoa deve se posicionar de modo que cubra toda área da imagem. Com isso, a pessoa está localizada em uma região adequada do cenário para que possa alcançar todas as áreas de interação que serão produzidas pelo jogo.

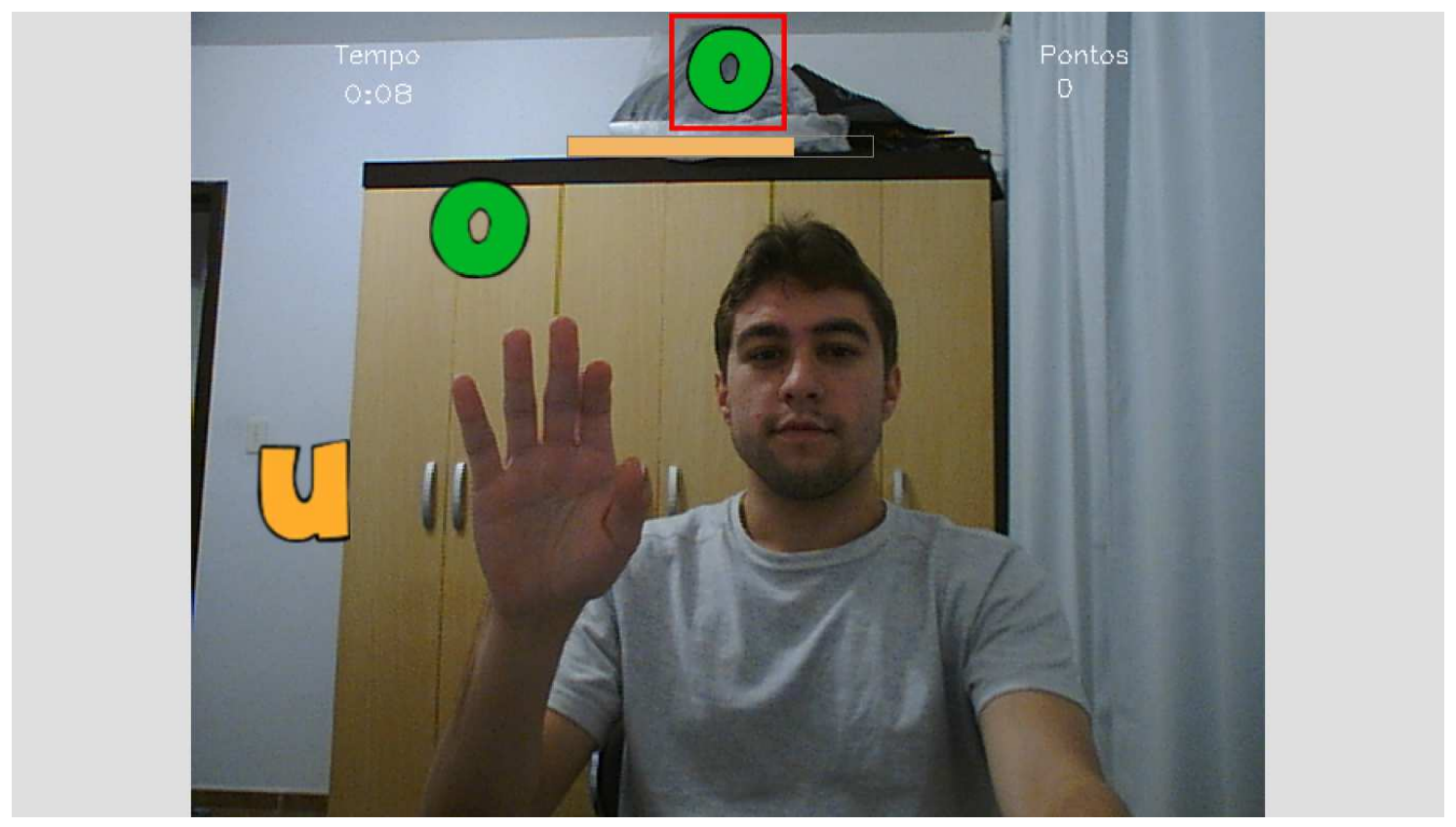

Figura 2: Imagem do cenário do MoviLetrando.

Após a calibração, o jogo inicia mostrando a imagem da webcam, ou seja, a própria pessoa se vê dentro do cenário do jogo não sendo necessário que o fundo seja uma superfície uniforme (é suficiente que não se tenha movimentos de pessoas, objetos nem das condições de iluminação). Também é gerado na parte superior do cenário, uma letra ou um número ditos referência e, ao mesmo tempo, é emitido um som representando este símbolo de referência. Logo abaixo, é mostrado um conjunto de símbolos, sendo um deles, igual ao da referência. O objetivo do jogador é usar o movimento dos braços e mãos para "tocar virtualmente" o símbolo correto. Entre o 
símbolo de referência e o conjunto de símbolos dispostos no cenário, existe uma barra que representa o tempo de exposição dos símbolos, ou seja, o tempo que o jogador tem para capturar um símbolo.

Um conceito útil no MoviLetrando é a lateralidade. O jogo pode limitar o jogador a usar apenas um dos braços, gerando os símbolos apenas no lado esquerdo, apenas no lado direito ou em ambos os lados. Esse comportamento do jogo é mudado conforme o jogador vai evoluindo na partida.

A pontuação do jogo é dada através dos acertos dos símbolos capturados e do tempo de exposição dos símbolos. Quanto mais rápido o jogador tocar nos símbolos, mais pontos irá ganhar para aquele acerto. Quando o jogador toca no símbolo igual a referencia, é emitido um retorno sonoro indicativo de acerto e quando ele erra ou perde o tempo, é emitido um som indicativo de erro. Caso o jogador não consiga pegar a tempo ou pegar o símbolo errado, ele não perde pontos, mas também não irá pontuar. Desta forma, a pontuação do jogo é sempre crescente, mais rápido ou mais lento conforme a prontidão em reconhecer e tocar o símbolo. Neste sentido, a pontuação adquire um significado diretamente relacionado com a evolução das habilidades que o próprio jogo está tentando desenvolver.

Durante uma partida, existe uma evolução nos níveis de dificuldade caso o jogador esteja demonstrando evolução (avaliado pela forma como adquire os pontos e pela própria pontuação):

- O tempo de exposição de todos os símbolos diminui;

- Em um determinado momento, a imagem de referência deixa de ser mostrada e apenas o som é emitido (quando então o jogador deverá ficar mais atento afim de identificar qual o símbolo correto da vez);

- Em fases avançadas, os símbolos mostrados poderão assumir formas diferentes das referências, forçando o jogador a analisar melhor para encontrar o símbolo correto;

- Da mesma forma que o formato varia, a cor dos símbolos também variam;

- Conforme o jogador evolui nos níveis, o número de símbolos gerados também aumenta, consequentemente, o tamanho dos símbolos diminui para poder caber todos os símbolos gerados;

- Outro aspecto que pode mudar em uma mesma partida, é a lateralidade.

A medida que o número de símbolos gerados aumenta, o jogador se depara com situações de ter que refletir e desenvolver movimentos menos óbvios para poder alcançar uma determinada letra que pode estar "atrás" de uma outra, tornando o jogo além de divertido, mais exigente quanto ao aspecto visio-motor.

Os níveis acima mudam automaticamente durante uma única partida. E as partidas podem ser configuradas antes de iniciar o jogo, mudando-se, por exemplo, o conjunto de símbolos usados no jogo: Vogais; Números; Vogais e Números; Consoantes; Vogais e Consoantes; Números e Consoantes; Vogais, Números e Consoantes. Além do conjunto e tamanho de símbolos, é possível determinar o tempo 
de duração de uma partida, conforme pode ser inferido pela Figura 3 que mostra a tela inicial do jogo.

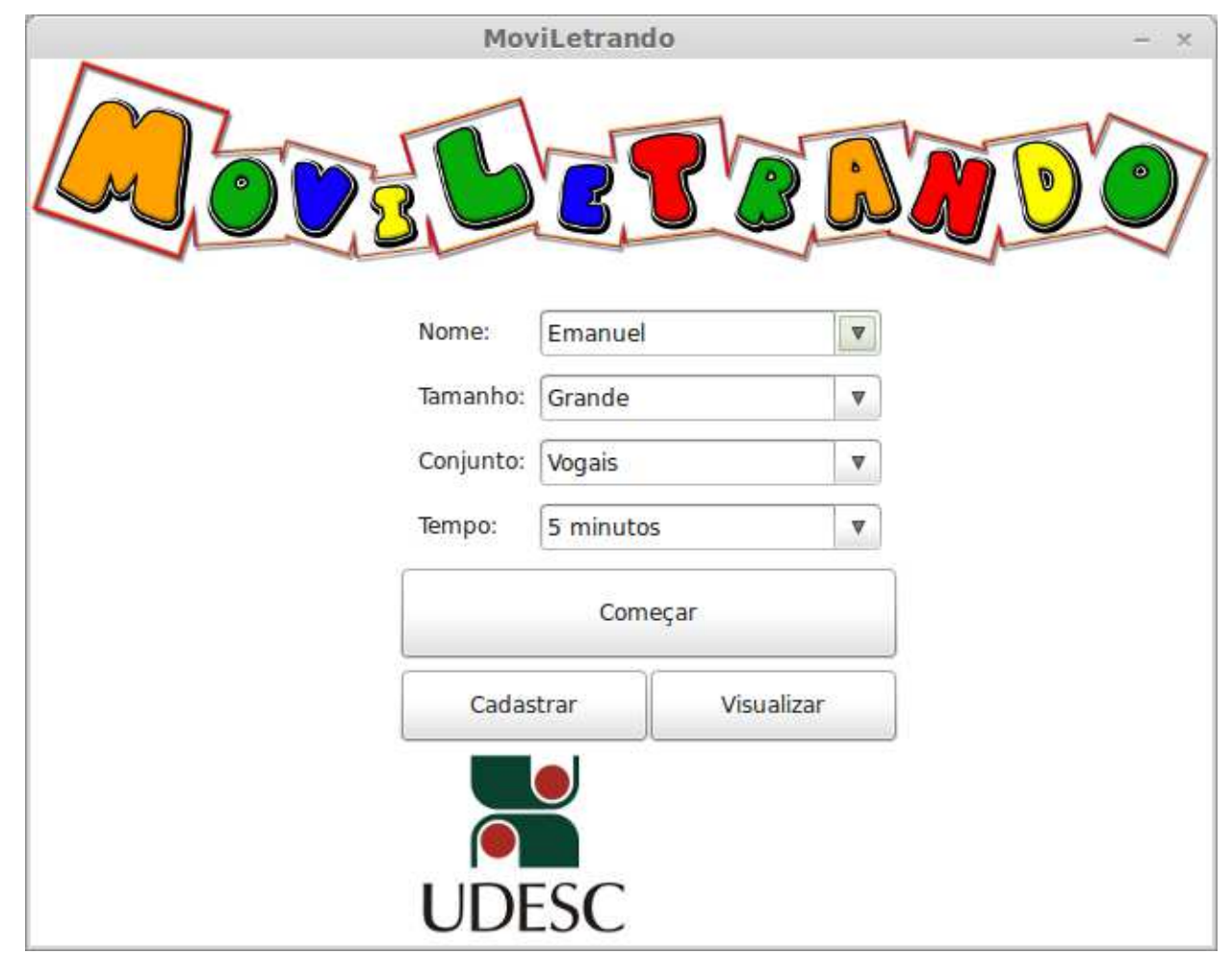

Figura 3. Imagem da tela inicial do MoviLetrando. Mostra as possíveis configurações antes de iniciar uma partida.

O jogo conta ainda com um banco de dados com informações a respeito das crianças. No final de cada partida são guardadas as informações a respeito da partida, acertos, erros, pontuação, etc. É possível ainda cadastrar várias crianças no banco. Com todos esses dados, é possível fazer uma análise a respeito da evolução de cada criança durante as diversas fases do jogo ao longo do período de estimulação determinado.

\section{Conclusão}

Apesar do surgimento dos Jogos Sérios, que focam em aspectos além do entretenimento como o educacional, por exemplo, ainda existem poucos Jogos Sérios que são desenvolvidos para deficientes intelectuais (DI). Alguns jogos comerciais que poderiam ser usados para desenvolvimento motor e cognitivo usam dispositivos que devem ser segurados pela criança, o que pode dificultar e desviar a atenção do foco principal do jogo. Estes aspectos se tornam mais significativos se considerarmos o público-alvo de crianças com DI.

Com base em experiências de outras pesquisas, pôde-se perceber que existem vantagens e benefícios para crianças com síndrome de Down usarem Jogos Sérios que se utilizam da tecnologia da RV de Projeção que capturam a imagem do jogador através de uma webcam, prescindindo de dispositivos para segurar e interagir. Em especial, 
percebeu-se também que o processo de letramento traz grandes benefícios de autoestima, independência e autonomia para esta população sendo pois, de grande valia.

Este artigo apresentou o MoviLetrando, um jogo para o letramento de crianças com síndrome de Down baseado em RV de Projeção que pode ser executado com um PC e Webcam convencionais (e baratos). O MoviLetrando consegue com a combinação dos estímulos sonoro, visual e motor, estimular o desenvolvimento motor e cognitivo, contribuindo para a coordenação motora, a propriocepção, a atenção/concentração, lateralidade, e reconhecimento pré-silábico de símbolos gráficos de letras.

Como trabalhos futuros, pretende-se testar o MoviLetrando com crianças assistidas pela rede pública de saúde para se determinar a sua aceitação e eficácia como recurso estimulante e/ou motivante no processo do letramento.

\section{Agradecimentos}

Os autores gostariam de agradecer ao Conselho Nacional de Desenvolvimento Científico e Tecnológico (CNPq) e à UDESC.

\section{Referências}

Annetta, L.A., Minogue, J., Holmes, S.Y. e Cheng, M.-T. (2009) Investigating the impact of video games on high school students' engagement and learning about genetics. Computers \& Education, 53, pp 74-85.

Baker, R.S.J.D, D’Mello, S. K., Rodrigo, M.M.T, Graesser, A. C. (2010). Better to be frustrated than bored: the incidence, persistence and impact of learners' cognitive affective states during interaction with three different computer-based learning environments. International Journal of Human-Computer Studies, 68, pp 223-241.

Bruckheimer, A. D.; Hounsell, M. S.; Kemczinski, A. Dance2Rehab: Um Jogo para Reabilitação Virtual Adaptativa. Anais: Simpósio Brasileiro de Jogos e Entretenimento Digital, 2010, Florianópolis - SC. IX SBGames (ISSN: 2179-2259), Novembro. Porto Alegre - RS: SBC, 2010. v. 1. p. 68-76.

Burke, J. W., Morrow, P. J., Mcneil M. D. J., Mcdonough, S. M., Charles, D. K. (2008) "Vision Based Games For Upper-Limb Stroke Rehabilitation". Anais: International Machine Vision and Image Processing Conference. IMVIP'08. Pp. 159-164

Cameirão, M.S., Bermúdez I., Badia, S., Oller, E., Zimmerli, L., and Verschure, P.F.M.J. (2007) The Rehabilitation Gaming System: A virtual reality based system for the evaluation and rehabilitation of motor deficits. Anais: Virtual Rehabilitation, ISBN 1-4244-1204-8, 27-29 September, Venice, Italy.

Deshpande, A. A. e Huang, S. H. Simulation Games in Engineering Education: A Stateof-the-Art Review. Computer Application in Engineering Education. DOI 10.1002/cae.20323. 2009.

EYETOY.

Disponível

em pt.playstation.com/ps3/peripherals/detail/item78905/PlayStation\%C2\%AEEye. Acessado em 09/08/2013. 
Farrington, J (2011) From the research: myths worth dispelling: seriously, the game is up. Performance Improvement Quaterly, 24, pp. 105-110.

FSD Fundação Síndrome de Down. Disponível em http://www.fsdown.org.br. Acessado em 07/08/2013.

Girard, C, Ecalle, J, Magnant, A. Serious Games as New Educational Tools: how effective are they? A meta-analysis of recent studies. Journal of Computer Assisted Learning. Jun 2012, pp 1-13. DOI: 10.1111/j.1365-2729.2012.00489.x

Gomes, A. L. L.; Fernandes, A. C.; Batista, C. A. M; Salustiano, D. A.; Mantoan, M. T. E.; Figueiredo, R. V.. Atendimento Educacional Especializado: deficiência mental. Brasília: SEESP/SEED/MEC, 2007.

GX - Gesture Extreme. Disponível em www.gesturetek.com. Acessado em 09/08/2013.

Marsh, T. (2011) Serious Games Continuum: Between games for purpose and experiential environments for purpose. Entertainment Computing, 2, pp 61-68.

Martin, S. Diaz, G. Sancistobal, E, Gil, R. Castro, M., Peire, J. (2011) New Technology trends in education: seven years of forecast and convergence. Computers \& Education, 57, 18993-1906.

Miller, L.M., Chang,C.-I., Wang, S., Beier, M. E., Klisch, Y (2011) Learning and Motivational Impacts of a multimedia science game. Computers \& Education, 57, 1425-1433.

Prensky, M. (ed.) Digital Natives, digital immigrants. In the Horizon, MCB University Press. 9, 2001, pp. 1-6.

Rizzo, A. "Skip". (2012) Clinical Virtual Reality: A Brief Review of the Future!. Anais: Symposium on Virtual and Augmented Reality. Keynote Speaker. Niterói (RJ):SBC. SVR 2012.

Rizzo, A. "Skip". Virtual Reality Definitions And Rationale For Its Use For Cognitive Assessment And Rehabilitation. Anais: World Congress on Brain Injury. Torino, Italy, May, 2001, pp 243-244.

Roizen, N. Down Syndrome. Anais: M.L. Batshaw (Ed.), Children with disabilities (5th ed.). Baltimore, ML : Paul H. Brookes, 2002.

Sacks, B.; Buckley, S. "What do we know about the movement abilities of children with Down syndrome". Down Sindrome News and Update. 2(4), Fev 2003, pp 131141.

Santamaria, V. L, Leitão, P. B, "A Consciência Fonológica no Processo de Alfabetização". http://www.cefac.br/revista/revista63/Artigo\%201.pdf. Acessado em 07/08/2013.

Sherman, W. R., Craig, A. B. Understanding Virtual Reality: Interface, Application, And Design. New York: Morgan Kaufmann, 2003, 582 p. ISBN 1558603530. 